\title{
Children with COVID-19 behaving milder may challenge the public policies: a systematic review and meta-analysis
}

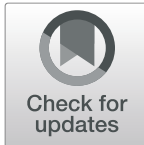

\author{
Chan $\mathrm{Liu}^{\dagger}, \mathrm{Yu} \mathrm{He}{ }^{\dagger}$, Lian Liư ${ }^{\dagger}$ Fang Li ${ }^{*}$ and Yuan Shi
}

\begin{abstract}
Background: The emerging virus is rampaging globally. A growing number of pediatric infected cases have been reported. Great efforts are needed to cut down the transmission.

Methods: A single-arm meta-analysis was conducted. We searched PubMed, Google Scholar, Web of Science, and several Chinese databases for studies presenting characteristics of children confirmed with Coronavirus Disease 2019 (COVID-19) from December 12, 2019 to May 10, 2020. Quality Appraisal of Case Series Studies Checklist was used to assess quality and publication bias was analyzed by Egger's test. Random-effect model was used to calculate the pooled incidence rate (IR) or mean difference (MD) with 95\% confidence intervals (Cl), or a fixed model instead when $\mathrm{I}^{2}<50 \%$. We conducted subgroup analysis according to geographic region. Additionally, we searched United Nations Educational Scientific and Cultural Organization to see how different countries act to the education disruption in COVID-19.
\end{abstract}

Results: 29 studies with 4300 pediatric patients were included. The mean age was 7.04 (95\% Cl: 5.06-9.08) years old. $18.9 \%$ of children were asymptomatic (95\% Cl: $0.121-0.266$ ), 37.4\% (95\% Cl: $0.280-0.474)$ had no radiographic abnormalities. Besides, a proportion of $0.1 \%$ patients were admitted to intensive care units (0, 95\% Cl: $0.000-0.013)$ and four deaths were reported (0,95\% Cl: 0.000-0.000). Up to 159 countries have implemented nationwide school closures, affecting over $70 \%$ of the world's students.

Conclusion: Children were also susceptible to SARS-CoV-2, while critical cases or deaths were rare. Characterized by mild presentation, the dilemma that children may become a potential spreader in the pandemic, while strict managements like prolonged school closures, may undermine their well-beings. Thus, the public policies are facing challenge.

Keywords: COVI-19, SARS-CoV-2, Children, Meta-analysis, School closure

\footnotetext{
* Correspondence: rematalili@ospital.cqmu.edu.cn; petshi530@vip.163.com

${ }^{\dagger}$ Chan Liu, Yu He and Lian Liu contributed equally to this work.

Department of Neonatology, Children's Hospital of Chongqing Medical

University; National Clinical Research Center for Child Health and Disorders;

Ministry of Education Key Laboratory of Child Development and Disorders,

China International Science and Technology Cooperation base of Child

Development and Critical Disorders; Chongqing Key Laboratory of Pediatrics,

Chongqing 400014, People's Republic of China
}

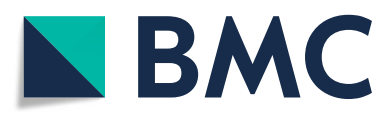

(c) The Author(s). 2020 Open Access This article is licensed under a Creative Commons Attribution 4.0 International License, which permits use, sharing, adaptation, distribution and reproduction in any medium or format, as long as you give appropriate credit to the original author(s) and the source, provide a link to the Creative Commons licence, and indicate if changes were made. The images or other third party material in this article are included in the article's Creative Commons licence, unless indicated otherwise in a credit line to the material. If material is not included in the article's Creative Commons licence and your intended use is not permitted by statutory regulation or exceeds the permitted use, you will need to obtain permission directly from the copyright holder. To view a copy of this licence, visit http://creativecommons.org/licenses/by/4.0/. The Creative Commons Public Domain Dedication waiver (http://creativecommons.org/publicdomain/zero/1.0/) applies to the data made available in this article, unless otherwise stated in a credit line to the data. 


\section{Background}

In December 2019, dozens of pneumonia cases with unknown etiology were reported in Wuhan, Hubei Province of China. Further sequencing analysis on samples of bronchoalveolar lavage fluid from pneumonia patients indicated that a new type of coronavirus, 2019 novel coronavirus (2019-nCoV), later renamed as severe acute respiratory syndrome coronavirus 2 (SARS-CoV-2), was to blame for this outbreak [1, 2]. The emerging disease caused by this pathogen, was then named Coronavirus Disease 2019 (COVID-19) officially by the World Health Organization (WHO). Human-to-human transmission has been recognized early onset of the spread of COVID-19 [3], and the numbers of confirmed cases keeps surging over the past few months. On 11 Mar 2020, the outbreak of COVID-19 was formally classified as a worldwide pandemic. As of 17 May, altogether 4,525,497 confirmed cases and 307,395 deaths across 215 countries were reported by WHO [4].

Though the SARS-CoV-2 is, based on current updated knowledge, phylogenetically, different from severe acute respiratory syndrome coronavirus (SARS-CoV) and Middle East Respiratory Syndrome-coronavirus (MERS $\mathrm{CoV}$ ), which were identified as the cause of the two previous epidemics occurred in China and Saudi Arabia, they do share certain similarities. SARS-CoV-2 shares $79 \%$ genome sequence similarity to SARS-CoV and 50\% genome sequence homology to MERS-CoV [5]. All the three viruses belong to Beta coronavirus and are enveloped positive-strand RNA viruses, patients got infected mainly manifested with respiratory symptoms (e.g. fever and cough) and poor clinical outcomes often associated with older age and underlying diseases $[5,6]$. Children with SARS or MERS appeared to develop a milder clinical course, thus resulted in a significant low mortality in the two previous outbreaks [7, 8]. An earlier study on 2143 pediatric patients by Dong [9] and colleagues found that $3 \%$ of laboratory-confirmed cases were severe/critical, while $7.4 \%$ in suspected cases.

So far, SARS-CoV-2 infection has aroused grave concern globally, however, it seems that children got less focused due to a milder presentation. Evidence-based data is in an urgent need to make up the gap in understanding clinical spectrum of COVID-19 in children. Therefore, we are going to synthesize and summarize the clinical characteristics and epidemiology of children with COVD-19 based on the latest literatures to provide a systematic view towards pediatric patients.

\section{Methods}

The protocol of this review followed recommendations established by Preferred Reporting Items for Systematic Reviews and Meta-Analyses (PRISMA) guidelines [10] and was registered in the International Prospective
Register of Systematic Reviews (PROSPERO) database (ID: CRD42020173233).

\section{Search strategy}

A systematic search was conducted in the following electronic databases: PubMed/Medline, Google Scholar, Web of Science, China National Knowledge Infrastructure (CNKI), Wanfang and several Chinese medical journals from December 1, 2019 to May 10,2020, incorporating the terms "COVID-19", "SARS-CoV-2", "children", "pediatric" etc. No language limitations were applied. The detailed search strategy can be found in Additional file 1.

Additionally, we searched United Nations Educational Scientific and Cultural Organization (UNESCO, https:// zh.unesco.org/) to find out how different countries act to the education disruption in COVID-19.

\section{Study selection}

The studies included in this meta-analysis should meet the following criteria: (1) all types of studies either retrospective or prospective (e.g. cohort, cross-sectional study, case report, case series); (2) studies reporting information regarding COVID-19; (3) studies describing clinical characteristics of pediatric patients (0-19 years) diagnosed by RT-PCR; (4) clinical data of more than five cases can be drawn from the articles. Duplicate studies were removed. Studies that select cases from the same hospital during the same period were excluded to avoid regional bias and potential redundant report, then articles with maximum cases were retained. We also excluded studies that reported data on both adults and children, where we failed to extract pediatric data.

\section{Data extraction}

Data were extracted from included studies by two reviewers (CL and LL) using Microsoft Excel 2019 independently, any disagreements were resolved by discussion with a third investigator $(\mathrm{YH})$. We extracted study characteristics including study design, time of enrollment, institutions, sample size, study subject features age, gender, epidemiology, symptoms and signs(e.g., fever, cough, lack of symptom), laboratory findings (e.g. white blood cell counts $[\mathrm{WBC}]$, lymphocyte counts [L],et.), radiographic images, treatments and outcomes(e.g. discharged, death). Primary presentation described in each study were extracted with no assumptions.

\section{Assessment of methodological quality}

Quality assessment of eligible studies was performed by the Quality Appraisal of Case Series Studies Checklist of the Institute of Health Economics (IHE) [11], which is comprised of 20 items. Each item would be scored ' 0 ' if it was answered 'NO' or 'UNCLEAR', if the answer was 'YES', the item scored ' 1 '. A study with 14 or more 
scores $(\geq 70 \%)$ was considered to be of acceptable quality.

\section{Statistical analysis}

The statistical software R 3.6.3 (R Foundation) was used to carry out the single-arm meta-analysis. Original data extracted from the literature will be transformed by the double arcsine method if the data is not normally distributed. Pooled incidence rates (IR) and 95\% confidence intervals $(95 \% \mathrm{CI})$ were calculated or dichotomous data and mean difference (MD) with $95 \% \mathrm{CI}$ were used to report continuous data. The $x^{2}$ test and the $\mathrm{I}^{2}$ statistic were used to assess heterogeneity among studies with the random-effect model and DerSimonian and Laird method, or a fixed model instead when $\mathrm{I}^{2}<50 \%\left(\mathrm{I}^{2}>50 \%\right.$ indicated that heterogeneity was statistically significant). We also conducted a subgroup analysis according to geographic region (Wuhan and outside Wuhan) to explore reasons for heterogeneity. In addition, a sensitivity analysis was followed by.

Publication bias was assessed using funnel plots and Egger's regression asymmetry test for meta-analysis that included at least 10 studies. $P$-value of $<0.05$ indicated the existence of publication bias.

\section{Results}

\section{Study search and characteristics}

A total of 1375 relevant papers were identified after a systematic search. (see Fig. 1). For those which were accessible to pediatric data, we conducted a comprehensive screening and comparison according to time of enrollment, institutions and demographic characteristics of subjects, 24 articles were under suspicion of an overlapped data were removed. Of 29 studies [12-40] incorporating 4300 children included in this meta-analysis, 20 were case series, 4 cross-sectional, 3 prospective cohorts and 2 retrospective cohort, none compared cases with controls. Study size ranged from 5 to 2572 participants from six countries (China, Italy, United States, Canada, Spain, Rome). The detailed characteristics can be found in Supplementary Table 1 (see Additional file 2).

As of 17 May 2020, 159 countries were reported to have implemented nationwide school closures to mitigate the impact of COVID-19 on children, affecting over 70\% of the world's student population.

\section{Demographical characteristics and epidemiology}

The mean age of pediatric patients enrolled in the 29 studies was 7.04 years old (95\% CI: 5.06-9.08), range from 1 day to 19 years old. Particularly, $12 \%$ (95\% CI: 0.063-0.188) of children were less than 1 year old, $14.9 \%$ (95\% CI:0.105-0.196) were 1 to 4 years old, $23.2 \%$ were 5 to 9 years, $23.1 \%$ were 10 to 14 years and $5.8 \%$ were more than 15 years old. Among them boys accounted for 53.6\% (95\% CI: 0.494-0.577). Comorbidities were reported in six studies with a proportion of $9.9 \%$ (95\% CI: 0.002-0.215).

A large number of cases were identified as part of family clusters with COVID-19, the pooled incidence rate was up to $81.5 \%$ (95\% CI:0.710-0.903). Besides, the pooled prevalence of cases associated with original epidemic area was 39.8\% (95\% CI:0.180-0.635).

\section{Severity of disease}

Fourteen studies described the severity of COVID-19 in pediatric group with 1 patient diagnosed as critical type $(0,95 \%$ CI:0.000-0.006) and 2 as severe type $(0,95 \%$ CI: 0.000-0.006).

\section{Clinical manifestations}

After a systematic review, we found 26 symptoms and signs reported in children infected with SARS-CoV-2. For the features of "sneezing", "swollen tonsils", "headache", "wheeze", "chill/rigor", "chest pain/distress", "abdominal pain", "seizure/convulsion", "rash", "constipation", "anosmia", "arthralgia", "conjunctivitis", "cyonosis" and "tachycardia", meta-analysis was thought to be unnecessary since few researches have presented. As is shown in Table 1, fever (52.7, 95\% CI: 0.443-0.610) and cough (41.9, 95\% CI:0.357-0.481) were the most prevalent and mild or moderate fever was more frequent than high fever. Lack of symptoms was also relatively common in these included cases, which turned out a proportion of 18.9\% (95\% CI: 0.121-0.266). Conversely, other symptoms or signs didn't have such a frequent presentation (summarized in Table 1).

\section{Laboratory findings}

The frequency of decreased WBC was similar to increased WBC in reported cases, the pooled incidence rate was 10.6\% (95\% CI: $0.054-0.168)$ and $10.3 \%$ (95\% CI: $0.066-$ 0.146). Compared to lymphopenia (10.8, $95 \%$ CI: 0.039 0.197), the incidence of lymphocytosis (15.4, 95\% CI: $0.098-0.217)$ was slightly higher in pediatric patients. Increased C-Reactive Protein (CRP) was in 12.3\% (95\% CI: $0.054-0.210$ ) of subjects. The pooled incidence rate of an elevated level of aspartate aminotransferase (AST), alanine aminotransferase (ALT) and lactate dehydrogenase (LDH) were $10.9 \%$ (95\% CI:0.050-0.182), 6.5\% (95\% CI:0.0380.096), 23.0\% (95\% CI:0.088-0.383) respectively.

\section{Radiographic findings}

Normal radiologic presentation was reported in 37.4\% (95\% CI: 0.280-0.474) of cases. Apart from that, the most common manifestation was ground-glass opacity (GGO) (35.7, 95\% CI:0.310-0.405), unilateral compromised lesions were more frequently presented than bilateral (28.2, 95\% CI:0.194-0.378 vs $21.9,95 \%$ CI: 0.104-0.355). Additionally, 


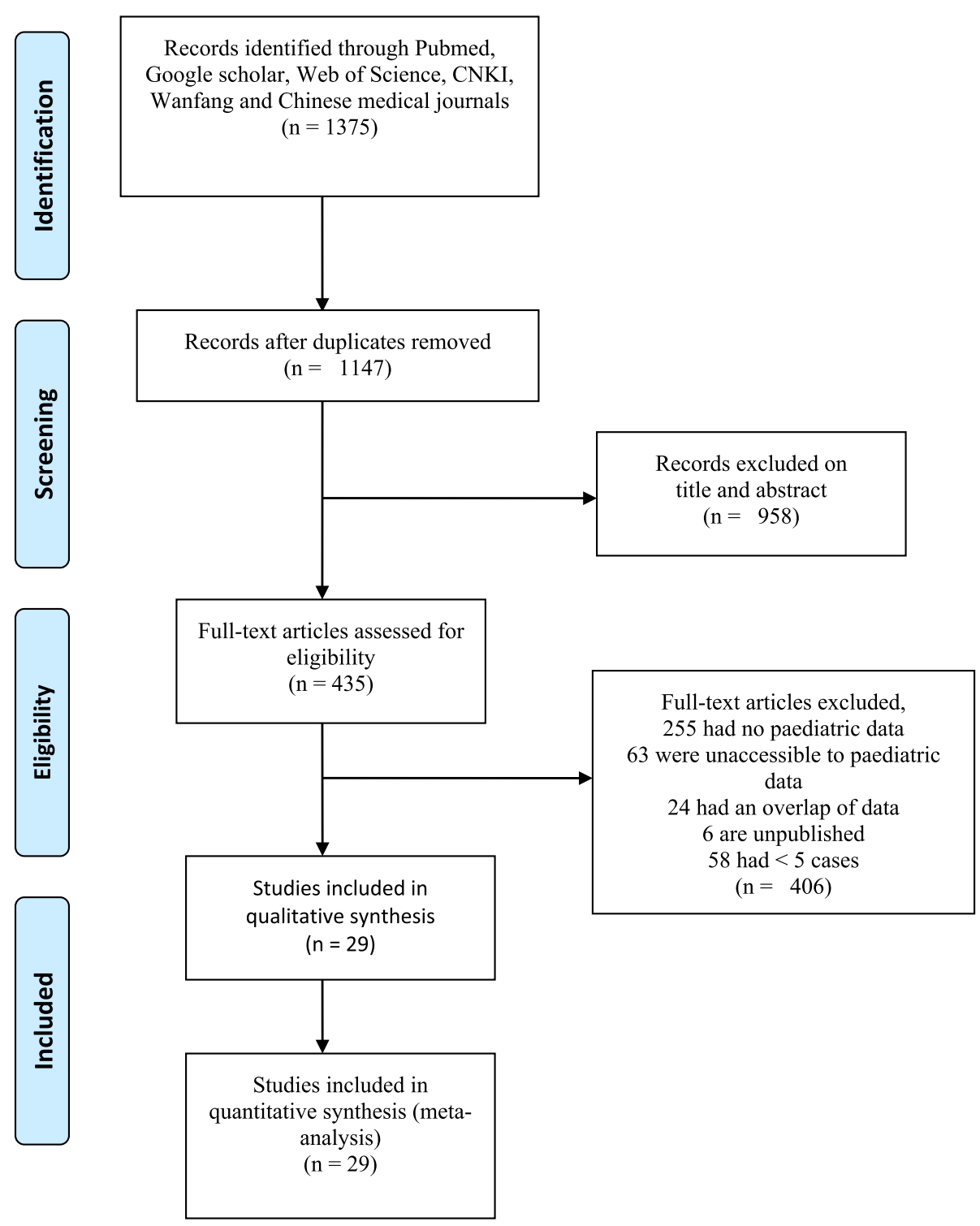

PRISMA 2009 flow diagram of the included studies

Fig. 1 PRISMA 2009 flow diagram of the included studies

10.5\% of subjects (95\% CI:0.016-0.236) were reported with consolidation on computed tomography (CT) imaging.

\section{Treatments and clinical outcomes}

Approximate 63.0\% (95\% CI: 0.255-0.939) SARS-CoV-2 infected children were treated with interferon and lopinavir-ritonavir (26, 95\% CI: 0.255-0.939) was more frequently applied compared with other antiviral agents including ribavirin, oseltamivir and arbidor. Few cases received administration of corticosteroid, immunoglobin therapy and mechanic ventilation, the pooled incidence rate was $0.0 \%$ (95\% CI: $0.0000-0.004), 0.0 \%$ (95\% CI: 0.000-0.003) and 0.0\%(95\% CI:0.000-0.002), respectively.
The majority of patients (84.1\%,95\% CI: 0.696-0.951) got discharged from hospital and $0.1 \%$ (95\% CI: 0.000-0.013) were transferred to intensive care units. Unfortunately, 4 deaths (0,95\% CI: 0.000-0.000) were ultimately confirmed.

\section{Subgroup analysis and sensitivity analysis}

The results of heterogeneity assessment and publication bias are shown in Table 1. Subgroup analysis indicated that geographic region may account for the heterogeneity of "fever (mild)" and "unilateral compromised". (Table 2). A further exploration for between-study heterogeneity by sensitivity analysis showed that none of these studies should be excluded. 
Table 1 Results of Meta-analysis on children with COVID-19

\begin{tabular}{|c|c|c|c|c|c|c|c|}
\hline Characteristics & Events/Total & $\mathrm{N}$ of studies & $\begin{array}{l}\text { Mean/Pooled } \\
\text { incidence (\%) }\end{array}$ & $95 \% \mathrm{Cl}$ & $\left.\right|^{2}(\%)$ & $P$-value & $\begin{array}{l}\text { Publication bias } \\
\text { ( } \boldsymbol{p} \text {-value) }\end{array}$ \\
\hline \multicolumn{8}{|l|}{ Demographic information } \\
\hline Male & $2305 / 4218$ & 29 & 53.6 & $49.4-57.7$ & 50 & $<0.01$ & 0.2161 \\
\hline Age (years) & $4300 / 4300$ & 29 & 7.04 & $5.06-9.08$ & 99.79 & $<0.01$ & $<0.05$ \\
\hline$<1$ & $602 / 4176$ & 24 & 12.0 & $6.3-18.8$ & 92 & $<0.01$ & 0.7211 \\
\hline $1-4$ & $456 / 3791$ & 21 & 14.9 & $10.5-19.6$ & 60 & $<0.01$ & $<0.05$ \\
\hline $5-9$ & $619 / 3791$ & 21 & 23.2 & $17.7-29.0$ & 67 & $<0.01$ & $<0.05$ \\
\hline $10-14$ & $960 / 3791$ & 21 & 23.1 & $21.6-24.6$ & 42 & 0.02 & 0.2341 \\
\hline $15-19$ & $1253 / 3962$ & 21 & 5.8 & $0.9-13.3$ & 95 & $<0.01$ & $<0.05$ \\
\hline Comorbidities & $159 / 927$ & 12 & 9.9 & $2.0-21.5$ & 94 & $<0.01$ & 0.5658 \\
\hline \multicolumn{8}{|l|}{ Epidemiology } \\
\hline Linkage to Wuhan & $275 / 465$ & 21 & 39.8 & $18.0-63.5$ & 95 & $<0.01$ & $<0.05$ \\
\hline Family cluster & $525 / 704$ & 20 & 81.5 & $71.0-90.3$ & 86 & $<0.01$ & 0.5347 \\
\hline \multicolumn{8}{|l|}{ Severity of disease } \\
\hline Mild \& Common & $344 / 347$ & 14 & 100.0 & $99.1-100.0$ & 0 & 1.00 & 0.4795 \\
\hline Severe & $2 / 347$ & 14 & 0 & $0.0-0.6$ & 0 & 1.0 & 0.0968 \\
\hline Critical & $1 / 347$ & 14 & 0 & $0.0-0.5$ & 0 & 1.0 & $<0.05$ \\
\hline \multicolumn{8}{|l|}{ Clinical manifestations } \\
\hline Asymptomatic & 248/1726 & 28 & 18.9 & $12.1-26.6$ & 86 & $<0.01$ & $<0.05$ \\
\hline Fever & $941 / 2017$ & 29 & 52.7 & $44.3-62.0$ & 87 & $<0.01$ & 0.1689 \\
\hline Mild $\left(37.7^{\circ} \mathrm{C}-38.0^{\circ} \mathrm{C}\right)$ & $72 / 426$ & 14 & 19.2 & $12.0-27.4$ & 56 & $<0.01$ & 0.0575 \\
\hline Moderate $\left(38.1^{\circ} \mathrm{C}-39.0^{\circ} \mathrm{C}\right)$ & $95 / 433$ & 15 & 15.5 & $9.0-22.9$ & 54 & $<0.01$ & 0.0819 \\
\hline $\operatorname{High}\left(39.1^{\circ} \mathrm{C}-\right)$ & $44 / 438$ & 15 & 8.2 & $2.8-15.3$ & 66 & $<0.01$ & 0.5681 \\
\hline Cough & $1035 / 2017$ & 29 & 41.9 & $35.7-48.1$ & 72 & $<0.01$ & $<0.05$ \\
\hline Expectoration & $14 / 270$ & 17 & 1.4 & $0.0-4.1$ & 43 & 0.03 & 0.464 \\
\hline Pharyngeal erythema & $105 / 429$ & 17 & 6.0 & $0.0-19.1$ & 91 & $<0.01$ & $<0.05$ \\
\hline Sore throat & $425 / 1985$ & 27 & 5.0 & $0.6-11.8$ & 93 & $<0.01$ & $<0.05$ \\
\hline Rhinorrhea & $455 / 1827$ & 27 & 3.5 & $0.1-9.8$ & 93 & $<0.01$ & $<0.05$ \\
\hline Stuffy nose & $26 / 592$ & 24 & 1.0 & $0.1-2.5$ & 46 & $<0.01$ & 0.4097 \\
\hline Diarrhea & $98 / 1021$ & 26 & 4.2 & $1.8-7.3$ & 45 & $<0.01$ & $<0.05$ \\
\hline Vomiting & $69 / 1021$ & 26 & 3.5 & $2.1-5.1$ & 35 & 0.04 & 0.0632 \\
\hline Tachypnea/dyspnea & $117 / 1057$ & 27 & 2.5 & $1.6-4.8$ & 72 & $<0.01$ & $<0.05$ \\
\hline Fatigue/myalgia & $103 / 1044$ & 25 & 2.7 & $0.3-6.4$ & 73 & $<0.01$ & $<0.05$ \\
\hline \multicolumn{8}{|l|}{ Laboratory findings } \\
\hline WBC decreased & $86 / 509$ & 20 & 10.6 & $5.4-16.8$ & 60 & $<0.01$ & 0.1130 \\
\hline WBC increased & $38 / 302$ & 18 & 10.3 & $6.6-14.6$ & 0 & 0.62 & 0.6663 \\
\hline $\mathrm{L}$ decreased & $56 / 497$ & 19 & 10.8 & $3.9-19.7$ & 80 & $<0.01$ & 0.131 \\
\hline$L$ increased & $33 / 182$ & 13 & 15.4 & $9.8-21.7$ & 43 & 0.05 & 0.7734 \\
\hline ALT increased & $39 / 405$ & 15 & 6.5 & $3.8-9.6$ & 43 & 0.04 & 0.3616 \\
\hline AST increased & $58 / 423$ & 14 & 10.9 & $5.0-18.2$ & 65 & $<0.01$ & 0.7864 \\
\hline LDH increased & $51 / 183$ & 13 & 23.0 & $8.8-38.3$ & 79 & $<0.01$ & 0.7704 \\
\hline CRP increased & $107 / 537$ & 18 & 12.3 & $5.4-21.0$ & 77 & $<0.01$ & 0.2760 \\
\hline \multicolumn{8}{|l|}{ Radiographic evaluation } \\
\hline Normal & 166/501 & 23 & 37.4 & $28.0-47.4$ & 77 & $<0.01$ & $<0.05$ \\
\hline
\end{tabular}


Table 1 Results of Meta-analysis on children with COVID-19 (Continued)

\begin{tabular}{|c|c|c|c|c|c|c|c|}
\hline Characteristics & Events/Total & $N$ of studies & $\begin{array}{l}\text { Mean/Pooled } \\
\text { incidence (\%) }\end{array}$ & $95 \% \mathrm{Cl}$ & $1^{2}(\%)$ & $\boldsymbol{P}$-value & $\begin{array}{l}\text { Publication bias } \\
\text { (p-value) }\end{array}$ \\
\hline GGO & $169 / 456$ & 19 & 35.7 & $31.0-40.5$ & 49 & $<0.01$ & 0.6935 \\
\hline Consolidation & $38 / 224$ & 14 & 10.5 & $1.6-23.6$ & 80 & $<0.01$ & 0.7874 \\
\hline Unilateral compromised & $93 / 365$ & 15 & 28.2 & $19.4-37.8$ & 55 & 0.01 & 0.0912 \\
\hline Bilateral compromised & $74 / 365$ & 15 & 21.9 & $10.4-35.5$ & 80 & $<0.01$ & 0.2329 \\
\hline \multicolumn{8}{|l|}{ Therapy } \\
\hline Oxygen therapy & $38 / 480$ & 17 & 4.9 & $2.7-7.5$ & 22 & 0.2 & 0.2031 \\
\hline \multicolumn{8}{|l|}{ Antiviral treatmen } \\
\hline Interferon & $140 / 370$ & 15 & 63.0 & $25.5-93.9$ & 98 & $<0.01$ & $<0.05$ \\
\hline Lopinavir-ritonavir & $68 / 216$ & 13 & 26.0 & $11.8-42.7$ & 83 & $<0.01$ & 0.5968 \\
\hline Ribavirin & $14 / 216$ & 13 & 2.9 & $0.5-6.4$ & 46 & 0.03 & 0.8658 \\
\hline Oseltamivir & $40 / 216$ & 13 & 10.5 & $0.5-27.2$ & 88 & $<0.01$ & 0.755 \\
\hline Arbidor & $35 / 216$ & 13 & 5.9 & $0.0-17.8$ & 82 & $<0.01$ & 0.1585 \\
\hline Antibiotics & $23 / 182$ & 13 & 11.3 & $1.8-25.4$ & 79 & $<0.01$ & 0.1121 \\
\hline Corticosteroid & $6 / 387$ & 16 & 0.0 & $0.0-0.4$ & 13 & 0.30 & $<0.05$ \\
\hline Immunoglobin & $8 / 381$ & 16 & 0.0 & $0.0-0.3$ & 55 & $<0.01$ & $<0.05$ \\
\hline Mechanic ventilation & $6 / 737$ & 23 & 0.0 & $0.0-0.2$ & 0 & 1.0 & 0.1143 \\
\hline \multicolumn{8}{|l|}{ Outcomes } \\
\hline Discharged & $419 / 553$ & 20 & 84.1 & $69.6-95.1$ & 92 & $<0.01$ & 0.7479 \\
\hline ICU admission & $32 / 1587$ & 28 & 0.1 & $0.0-1.3$ & 51 & $<0.01$ & 0.1641 \\
\hline Death & $4 / 4278$ & 14 & 0.0 & $0.0-0.0$ & 0 & 1 & $<0.05$ \\
\hline
\end{tabular}

Note: $W B C$ white blood cell counts, $L$ lymphocyte counts, $A S T$ aspartate aminotransferase, $A L T$ alanine aminotransferase, $L D H$ lactate dehydrogenase, $C R P C$ reactive protein, GGO ground-glass opacity, ICU intensive care unit

If the observed index wasn't reported in a research, 0 cases were calculated as occurred

Linkage to Wuhan referred to children who resided in Wuhan or travelled to Wuhan or contacted with people from Wuhan before the onset of infection Family cluster was defined as more than one infected family member residing with the child

\section{Discussion}

The unpredictable emergency of SARS-CoV-2 has posed a substantial threat to public health. Implementing efforts on aggregating the existing data about epidemiology, clinical, laboratory, and imaging characteristics to have a better understanding of the virus, its patterns of spread and the spectrum of illness is of critical significance. Through a comprehensive searching, a total of 29 articles with 4300 cases were included.

The proportion of male to female of this analysis $(53.6 \%$ vs $46.4 \%)$ is similar to the gender distribution in an initial investigation [9](57.5\% vs $42.5 \%$ in 731 confirmed cases) and general population [41] (55.9\% vs 44.1\%) (Table 3). All the results seem to show that male have a slightly higher incidence than female in COVID-19.

According to our results, children got infected with SARS-CoV-2 mainly through family clustering, quite the same as SARS-CoV [7] and MERS-CoV [8].While compared to adults, children are more likely to be asymptomatic or present with milder symptoms, this reminds us that, whenever there's a family member caught with this virus, it is necessary to conduct a virologic screening test on the child as soon as possible. Otherwise, the infected child may become a threat to other vulnerable populations (e.g. elderly people or people with severe underlying disease), resulting in further extension of ongoing pandemic, as was seen during influenza outbreak [44].

Fever and cough are the most common symptoms in COVID-19 children, in our study, the pooled incidence of fever is $52.7 \%$, which is lower than that in adults [41], SARS [7, 42] and influenza [43]. Clearly, children with COVID-19 rarely had obvious signs and symptoms of upper respiratory tract (pharyngeal congestion, rhinorrhea, sore throat, stuffy nose). Through a comprehensive review, it's easy to draw the conclusion that SARS-CoV-2 leads to a less aggressive clinical course in children with more asymptomatic and fewer symptoms, compared to that in adults and the other two pathogens. (Table 3 ).

In terms of laboratory abnormalities, only $10.8 \%$ of infected children presented with lymphopenia, which is quite different from findings in COVID-19 adults [41] and SARS [7].Besides, leucopenia was found in $10.6 \%$ of patients, nevertheless, a research including 80 virologicconfirmed children cited by Henry [45] reported $46 \%$ of lymphopenia. Theoretically, virus particles primarily spread through the respiratory mucosa, initially using 
Table 2 Subgroup analysis on the characteristics of children with COVID-19

\begin{tabular}{|c|c|c|c|c|c|c|}
\hline \multirow[t]{2}{*}{ Characteristics } & \multicolumn{3}{|l|}{ Wuhan } & \multicolumn{3}{|l|}{ Outside Wuhan } \\
\hline & $\mathrm{R}(95 \% \mathrm{Cl})$ & 12 & $\boldsymbol{p}$-value & $\mathrm{R}(95 \% \mathrm{Cl})$ & 12 & $\boldsymbol{p}$-value \\
\hline \multicolumn{7}{|l|}{ Demographic information } \\
\hline Male & $43.5(17.3-71.6)$ & $69 \%$ & 0.04 & $54.8(53.2-56.5)$ & $49 \%$ & $<0.01$ \\
\hline \multicolumn{7}{|l|}{ Age } \\
\hline$<1 \mathrm{y}$ & $38.7(0.0-96.5)$ & $94 \%$ & $<0.01$ & $9.4(4.1-16.1)$ & $91 \%$ & $<0.01$ \\
\hline $1-4 y$ & $31.8(0.0-1.0)$ & $93 \%$ & $<0.01$ & 8.8(7.7-9.9) & $48 \%$ & 0.01 \\
\hline $5-9 y$ & $3.5(0.0-22.8)$ & $35 \%$ & 0.22 & $24.5(18.8-30.5)$ & $69 \%$ & $<0.01$ \\
\hline $10-14 y$ & $0.0(0.0-12.4)$ & $0 \%$ & 0.90 & $23.3(21.8-24.8)$ & $31 \%$ & 0.09 \\
\hline $15-19 y$ & $0.0(0.0-12.4)$ & $0 \%$ & 0.00 & $8.7(3.3-15.7)$ & $90 \%$ & $<0.01$ \\
\hline Comorbidities & $0.0(0.0-1.6)$ & $0 \%$ & 0.80 & $12.6(3.5-25.4)$ & $92 \%$ & $<0.01$ \\
\hline \multicolumn{7}{|l|}{ Severity of illness } \\
\hline Mild \& Common & - & NA & NA & $1.0(99.1-1.0)$ & $0 \%$ & 0.99 \\
\hline Severe & - & NA & NA & $0.0(0.0-0.6)$ & $0 \%$ & 1.00 \\
\hline Critical & - & NA & NA & $0.0(0.0-0.5)$ & $0 \%$ & 1.00 \\
\hline \multicolumn{7}{|l|}{ Epidemiology } \\
\hline Linkage to Wuhan & $96.4(70.4-1.0)$ & $78 \%$ & 0.01 & $29.7(21.0-39.0)$ & $54 \%$ & $<0.01$ \\
\hline Family cluster & $92.5(87.6-0.965)$ & $0 \%$ & 0.33 & $79.2(68.0-88.8)$ & $83 \%$ & $<0.01$ \\
\hline \multicolumn{7}{|l|}{ Clinical manifestations } \\
\hline Asymptomatic & 19.1(13.0-25.9) & $17 \%$ & 0.30 & 19.9(12.2-28.6) & $86 \%$ & $<0.01$ \\
\hline Fever & $67.0(25.9-97.8)$ & $83 \%$ & $<0.01$ & $51.6(42.5-60.7)$ & $87 \%$ & $<0.01$ \\
\hline Mild $\left(37.7^{\circ} \mathrm{C}-38.0^{\circ} \mathrm{C}\right)$ & $6.2(2.4-11.2)$ & $0 \%$ & 0.54 & $20.8(15.5-26.5)$ & $23 \%$ & 0.21 \\
\hline Moderate $\left(38.1^{\circ} \mathrm{C}-39.0^{\circ} \mathrm{C}\right)$ & 19.1(13.0-25.9) & $17 \%$ & 0.30 & $15.1(7.0-24.9)$ & $60 \%$ & $<0.01$ \\
\hline $\operatorname{High}\left(39.1^{\circ} \mathrm{C}-\right)$ & $54.7(0.0-1.0)$ & $96 \%$ & $<0.01$ & $5.9(2.7-9.8)$ & $0 \%$ & 0.48 \\
\hline Cough & $61.5(23.2-93.7)$ & $81 \%$ & $<0.01$ & $40.2(33.7-46.8)$ & $73 \%$ & $<0.01$ \\
\hline Expectoration & $14.3(0.0-51.7)$ & NA & NA & $1.3(0.0-3.9)$ & $44 \%$ & 0.03 \\
\hline Pharyngeal erythema & $38.1(3.9-80.0)$ & $84 \%$ & $<0.01$ & $2.3(0.0-10.7)$ & $80 \%$ & $<0.01$ \\
\hline Sore throat & $0.0(0.0-0.0)$ & $0 \%$ & 0.56 & $6.2(1.4-13.0)$ & $91 \%$ & $<0.01$ \\
\hline Rhinorrhea & $4.9(1.5-9.5)$ & $0 \%$ & 0.45 & $3.8(0.0-11.6)$ & $93 \%$ & $<0.01$ \\
\hline Stuffy nose & $1.8(0.0-5.4)$ & $0 \%$ & 0.94 & $1.6(0.0-5.1)$ & $51 \%$ & $<0.01$ \\
\hline Diarrhea & $4.8(1.4-9.5)$ & $0 \%$ & 0.70 & $4.1(1.4-7.7)$ & $51 \%$ & $<0.01$ \\
\hline Vomiting & $14.4(0-52.9)$ & $83 \%$ & $<0.01$ & $3.4(1.9-5.1)$ & $18 \%$ & 0.22 \\
\hline Tachypnea/dyspnea & 16.4(1.6-38.6) & $55 \%$ & 0.11 & $4.3(2.7-6.1)$ & $47 \%$ & $<0.01$ \\
\hline Fatigue/myalgia & $4.5(1.3-9.1)$ & $0 \%$ & 0.65 & $2.6(0.1-6.8)$ & $75 \%$ & $<0.01$ \\
\hline \multicolumn{7}{|l|}{ Laboratory findings } \\
\hline WBC decreased & $39.6(6.6-78.4)$ & $74 \%$ & 0.05 & $8.4(5.2-12.2)$ & $26 \%$ & 0.15 \\
\hline WBC increased & $0.0(0.0-26.8)$ & NA & NA & 10.7(6.9-15.1) & $0 \%$ & 0.62 \\
\hline$L$ decreased & $48.4(0.0-1.0)$ & $97 \%$ & $<0.01$ & $8.8(3.0-16.5)$ & $68 \%$ & $<0.01$ \\
\hline$L$ increased & $0.0(0.0-26.8)$ & NA & NA & $16.3(10.5-22.8)$ & $43 \%$ & 0.06 \\
\hline ALT increased & $9.9(5.2-15.5)$ & $0 \%$ & 0.57 & $4.2(1.4-8.0)$ & $41 \%$ & 0.06 \\
\hline AST increased & $33.4(0.0-86.0)$ & $86 \%$ & $<0.01$ & $8.9(3.1-16.7)$ & $60 \%$ & $<0.01$ \\
\hline LDH increased & $33.3(1.3-76.4)$ & NA & NA & $21.4(7.8-38.5)$ & $80 \%$ & $<0.01$ \\
\hline CRP increased & 45.9(0.0-98.9) & $90 \%$ & $<0.01$ & $9.6(2.7-19.0)$ & $76 \%$ & $<0.01$ \\
\hline \multicolumn{7}{|l|}{ Radiographic evaluation } \\
\hline Normal & 13.1(7.7-19.3) & $0 \%$ & 0.62 & $39.7(29.8-50.1)$ & $66 \%$ & $<0.01$ \\
\hline
\end{tabular}


Table 2 Subgroup analysis on the characteristics of children with COVID-19 (Continued)

\begin{tabular}{|c|c|c|c|c|c|c|}
\hline \multirow[t]{2}{*}{ Characteristics } & \multicolumn{3}{|l|}{ Wuhan } & \multicolumn{3}{|l|}{ Outside Wuhan } \\
\hline & $\mathrm{R}(95 \% \mathrm{Cl})$ & 12 & $\boldsymbol{p}$-value & R (95\% Cl) & 12 & $\boldsymbol{p}$-value \\
\hline GGO & $30.9(23.6-38.6)$ & $0 \%$ & 0.67 & $38.1(29.1-47.5)$ & $51 \%$ & $<0.01$ \\
\hline Consolidation & $0.0(0.0-31.7)$ & NA & NA & 11.4(1.9-25.3) & $82 \%$ & $<0.01$ \\
\hline Unilateral compromised & $13.6(3.6-27.3)$ & $18 \%$ & 0.27 & $30.6(23.6-37.9)$ & $40 \%$ & 0.07 \\
\hline Bilateral compromised & 38.0(0.0-98.7) & $90 \%$ & $<0.01$ & 20.3(7.7-36.0) & $78 \%$ & $<0.01$ \\
\hline \multicolumn{7}{|l|}{ Therapy } \\
\hline \multicolumn{7}{|l|}{ Antiviral treatment } \\
\hline Interferon & 100.0(73.2-100.0) & NA & NA & $59.5(21.5-92.5)$ & $94 \%$ & $<0.01$ \\
\hline Lopinavir-ritonavir & $0.0(0.0-26.8)$ & NA & NA & $28.5(13.6-45.8)$ & $83 \%$ & $<0.01$ \\
\hline Ribavirin & $33.3(1.3-76.4)$ & NA & NA & $2.6(0.4-6.0)$ & $39 \%$ & 0.08 \\
\hline Oseltamivir & 100.0(73.2-100.0) & NA & NA & $6.1(0.0-19.2)$ & $85 \%$ & $<0.01$ \\
\hline Arbidor & $0.0(0.0-26.8)$ & NA & NA & $6.5(0.0-19.3)$ & $84 \%$ & $<0.01$ \\
\hline Antibiotics & 100.0(73.2-100.0) & NA & NA & $6.5(0.8-15.2)$ & $61 \%$ & $<0.01$ \\
\hline Corticosteroid & $66.7(23.6-98.7)$ & NA & NA & $0.0(0.0-0.2)$ & $0 \%$ & 1.00 \\
\hline Immunoglobin & $16.7(0.0,58.6)$ & NA & NA & $0.5(0.0-3.8)$ & $54 \%$ & $<0.01$ \\
\hline Mechanic ventilation & $0.0(0.0-1.4)$ & $0 \%$ & 0.96 & $0.0(0.0-0.2)$ & $0 \%$ & 1.00 \\
\hline \multicolumn{7}{|l|}{ Outcome } \\
\hline Discharged & $90.3(84.7-94.9)$ & $0 \%$ & 0.38 & 82.6(64.4-95.9) & $92 \%$ & $<0.01$ \\
\hline ICU admission & $0.5(0.0-7.5)$ & $28 \%$ & 0.25 & $0.1(0.0-1.4)$ & $53 \%$ & $<0.01$ \\
\hline Death & $0.0(0.0-0.2)$ & 0 & 0.81 & $0.0(0.0-0.0)$ & $0 \%$ & 0.97 \\
\hline
\end{tabular}

Note: $W B C$ white blood cell counts, $L$ lymphocyte counts, $A S T$ aspartate aminotransferase, $A L T$ alanine aminotransferase, $L D H$ lactate dehydrogenase, $C R P C$ reactive protein, GGO ground-glass opacity, ICU intensive care unit NA: not applicable, only one or no study included in the subgroup

Table 3 Comparison of incidence of clinical characteristics between children with COVID-19, general population with COVID-19, children with SARS and children with H1N1 influenza

\begin{tabular}{|c|c|c|c|c|}
\hline & Children with COVID-19 & $\begin{array}{l}\text { General population with } \\
\text { COVID-19 [41] }\end{array}$ & $\begin{array}{l}\text { Children with } \\
\text { SARS [42] }\end{array}$ & $\begin{array}{l}\text { Children with H1N1 } \\
\text { influenza [43] }\end{array}$ \\
\hline Age(y-old) & $5.5(3.44-7.65)$ & $51.97(46.06-57.89)$ & 12.2 & 5 \\
\hline Male & $53.6 \%(49.4-57.7)$ & $55.9 \%(51.6-60.1)$ & $45.5 \%$ & $54.7 \%$ \\
\hline Asymptomatic & $18.9 \%(12.1-26.6)$ & - & 0 & $<6.1 \%$ \\
\hline Fever & $52.7 \%(44.3-61.0)$ & $88.7 \%(84.5-92.9)$ & $100 \%$ & $93.9 \%$ \\
\hline Cough & $41.9 \%(35.7-48.1)$ & $57.6 \%(40.8-74.4)$ & $63.6 \%$ & $88.5 \%$ \\
\hline Sore throat & $5.0 \%(0.6-11.8)$ & $11.0 \%(2.8-19.2)$ & $13.6 \%$ & $19.6 \%$ \\
\hline Diarrhea & $4.2 \%(1.8-7.3)$ & $6.1 \%(2.4-9.7)$ & $20.5 \%$ & $6.1 \%$ \\
\hline Tachypnea/Dyspnea & $2.5 \%(1.6-4.8)$ & $45.6 \%(10.9-80.4)$ & $9.1 \%$ & - \\
\hline Leucopenia & $10.0 \%(4.5-16.7)$ & $18.7 \%(8.5-28.8)$ & $34.1 \%$ & $16.9 \%$ \\
\hline Lymphopenia & $10.8 \%(3.9-19.7)$ & $43.1 \%(18.9-67.3)$ & $77.3 \%$ & $34.5 \%$ \\
\hline Ground-glass opacity & $35.7 \%(31.0-40.5)$ & $68.5 \%(51.8-85.2)$ & - & - \\
\hline Comorbidities & $3 \%(11 / 361)$ & $36.8 \%(24.7-48.9)$ & $11.4 \%$ & $14.9 \%$ \\
\hline ICU admission & $0.1 \%(0.0-1.3)$ & $20.3 \%(10.0-30.6 \%)$ & $11.4 \%$ & $19.6 \%$ \\
\hline Death & $0(0.0-0.0)$ & $13.9 \%(6.2-21.5)$ & 0 & $2 \%$ \\
\hline$N$ & 4300 & 2874 & 44 & 148 \\
\hline
\end{tabular}

Note: The results of characteristics of COVID-19 in children and general population were presented with pooled incidence and $95 \%$ Cl, characteristics of "comorbidities" in children with COVID-19 were presented with incidence(n/N) due to insufficient data

No meta-analysis results of characteristics of children with SARS and H1N1 influenza were found, incidence $(\mathrm{n} / \mathrm{N})$ was presented as a substitute

"-": not available 
the angiotensin-converting enzyme 2(ACE2) receptor (the cell-entry receptor for SARS-CoV-2) at ciliated bronchial epithelial cells and infect other cells, induce a cytokine storm in the body, generate a series of immune responses, and cause changes in peripheral white blood cells and immune cells such as lymphocytes [46, 47]. Presumptions have been made that children may be protected against SARS-CoV-2 because this enzyme is less mature at a younger age, since the immune system undergoes substantial changes from birth to adulthood. In general, WBC and lymphocyte remained normal in the majority of pediatric patients, suggesting that the newly emerging virus, SARS-CoV-2, may have a marginal influence on the immune function of children.

As for radiologic aspects, our research found that a proportion of $37.4 \%$ of 501 virologic positive cases were in absence of CT abnormalities, and Ground glass opacity, also typical signs of severe acute respiratory syndrome (SARS) [7], was shown in $35.7 \%$ of pediatric patients. This kind of low sensitivity hints us that routinely radiologic scans should not be overemphasized for screening or early identification of COVID-19 in children in consideration of substantial radiation exposure, especially when the child is lack of symptoms or running a mild clinical course. Therefore, more strict strategies and screening practices are required for the better management of pediatric cases.

Compared to adults [41], the spread of SARS-CoV-2 yield a much better prognosis in pediatric patients, similar to SARS [7] and Middle East Respiratory Syndrome (MERS) [8]. 84.1\% of cases were discharged, the discharge rate ought to be higher actually since many children were still in hospital before the submission of the papers. The reasons why children experience a milder COVID-19 disease remain elusive. One possible explanation is that the response of children to SARS$\mathrm{CoV}-2$ is fundamentally different from that of adults, as demonstrated in earlier reports [48], the frequency of lymphopenia found in adults suggests that SARS-CoV-2 might act on lymphocytes, which is rare in children. Prior exposure to other respiratory virus may exert an influence, making children's immune systems more resilient [7]. Besides, some researchers proposed that the mild disease in children may be associated with trained immunity, which refers to the use of certain vaccines such as Bacille de Calmette Guerin (BCG). BCG has been proved to provide nonspecific protection of mice against influenza virus infection probably by the induction of trained immunity [44]. In addition, the virulence and pathogenicity of the virus may decrease in pediatric patients who are usually belong to the second or third generation infection. Accordingly, further studies in fields of immunology, anatomy and virology are required to ravel out this puzzle.
With massive public health interventions implemented actively and effectively, the spread of SARS-CoV-2 seems to have been under control in several countries. On 17 May 2020, there were only 7 newly confirmed cases across mainland China and 4 were imported [49]. At present, while some countries are considering enhancing control measures, China is planning to lift restrictions, work resumes and school starts are on agenda. Nevertheless, concerns have been proposed that a second wave of cases might occur in light of the absence of herd immunity against COVID-19, escalating case importation or residual infected seeds and resumption of economic activities [50, 51]. It's plausible to suggest whether children have to get away from school again to mitigate the revival transmission. School closures can affect the spread of virus during a pandemic through reducing transmission and new cases, while long periods of social distancing interventions in school may put students in a disadvantaged situation. Recently, some scholars are questioning the benefits brought by closing schools. On the one hand, school closures are based on empirical evidence and assumptions from influenza outbreaks, it's hard to say such measures are also effective in coronavirus outbreaks like SARS, MERS and especially COVID-19, for which transmission dynamics appear to be different [52]. A systemic review [52] concluded that school closures in SARS did not contribute to the control of the epidemic and its effectiveness in COVID-19 would be less than other social distancing interventions, with only $2-4 \%$ of death prevention. Meanwhile, less comprehensive and deliberate plan can result in a completely converse consequence. Jude Bayham and Eli P Fenichel [53] estimated that school closures could lead to mortality rate increased by $0.35 \%$ and a greater number of deaths than they prevent when the health-care workforce declines by $15.0 \%$ due to unintended childcare obligations. (Table 4 shows alternative closure strategies in five countries). On the other hand, prolonged school dismissals can be detrimental to children's physical and mental health $[59,60]$. Out of school means a totally altered lifestyle-for example, fewer physical activities, less interaction with peer groups and longer screen time. Besides, many schools are offering online courses, but this is not available to all, especially to children from low socioeconomic households, and they may be further disadvantaged by nutrition shortfalls. Moreover, with home confinement, communities lockdown and economic recession deepens, family conflicts are rising, children are more likely to be exposed to domestic violence and abuse. Consequently, it is imperative for the policy makers to weigh the benefits of school closure against its costs carefully and deliberately and provide alternative strategies to minimize the adverse impacts of the COVID-19 on children's well-being. 
Table 4 School strategies in different countries in response to COVID-19

\begin{abstract}
UK [54] Localized closures have been implemented since 28 Feb. All educational settings are closed to everyone except the children of critical workers and vulnerable children ${ }^{\mathrm{a}}$ since 20 March and will stay closed until further notice.

US [55] School-based strategies (e.g., short-term or extended dismissals, event cancellations, social distancing measures) are adopted locally in collaboration with local health officials based on level of community transmission of COVID-19 and presence of COVID-19 cases within the school, combined with open child care programs ${ }^{b}$ like private child care centers for essential service providers. The majority of States have mandated school closures since 10 April, including until the end of the academic year in June. Some States, however, have recommended but not mandated the school closures.

Italy [56] Some schools in the heaviest hit area have been shut down since 24 Feb. Mandatory closure of all schools and universities across the country were implemented from 10 March and will remain shut until 3 May.

France [57] All nurseries, schools, colleges, high schools and universities are closed from 16 March and will gradually reopen from 11 May with the exception of universities, which will not reopen until the summer. Childcare services are established for staff who are essential to the management of the health crisis.

German [58] Temporarily closing kindergartens and schools and postponing restart of colleges were implemented in state levels since mid-March are to be extended until 3 May 2020. Schools remain open for those who are willing to continue classes in some states. Daycare centres are available and will continue and will be extended to other occupational and needed groups.

Note: ${ }^{2}$ Vulnerable children include children who are supported by social care, those with safeguarding and welfare needs, including child in need plans, on child protection plans, 'looked after' children, young carers, disabled children and those with education, health and care (EHC) plans

${ }^{b}$ Other open child care programs are home-based child care, pre-kindergarten programs, Head Start and Early Head Start programs, temporary child care centers, and child care centers that partner with healthcare facilities to support healthcare workers who need child care
\end{abstract}

There are several limitations need to be acknowledged. Firstly, most of articles included in this meta-analysis are descriptive and retrospective with wide range of sample size, which highlighted different aspects of the illness, consequently, high heterogeneity was inevitable. Secondly, reports derived from China dominated the largest part, data from other countries are still in short. In addition, we have intended to conduct a subgroup analysis based on age stratification and severity of the disease, while enough information was unavailable. Therefore, the findings of this meta-analysis still need to be updated by more relevant studies with more strict design and larger sample size.

\section{Conclusions}

In conclusion, our study highlights the epidemiology, clinical characteristics of COVID-19 in pediatric patients. This quantitative analysis provides evidence-based knowledge for the diagnosis and management in pediatric patients in the ongoing pandemic. Children were also susceptible to SARS-CoV-2. Compared to adults, children experienced a milder clinical course. The most frequent symptoms were fever and cough, asymptomatic were also quite common. Children with no or mild symptoms should be virologic-screened and isolated from immunocompromised populations at once when a family member is diagnosed with COVID-19 to prevent child-driven transmission. A group of children were absent from CT abnormalities, CT scans should not be overemphasized to avoid excessive radiation exposure. Public health officials should attach importance to additional childcare programs to protect the wellbeing of children in this pandemic context.

\section{Supplementary information}

Supplementary information accompanies this paper at https://doi.org/10. 1186/s12887-020-02316-1.

Additional file 1. SEARCH STRATEGY: This file describes the search strategy of this meta-analysis

Additional file 2: Table S1. Characteristics of the included studies

\section{Abbreviations}

2019-nCoV: 2019 novel coronavirus; ACE2: Angiotensin-converting enzyme 2; ALT: Alanine aminotransferase; AST: Aspartate aminotransferase; BCG: Bacille de Calmette Guerin; COVID-19: Coronavirus Disease 2019; CRP: C-Reactive Protein; CT: Computed tomography; GGO: Ground-glass opacity; IHE: Institute of Health Economics; L: Lymphocyte counts; MERS: Middle East Respiratory Syndrome; MERS-CoV: Middle East Respiratory Syndrome-coronavirus; SARS: Severe acute respiratory syndrome; SARS-COV: Severe acute respiratory syndrome coronavirus; SARS-CoV-2: Severe acute respiratory syndrome coronavirus 2; WBC: White blood cell counts; WHO: World Health Organization

\section{Acknowledgements \\ Not applicable.}

\section{Authors' contributions}

YS generated the idea, designed the study and participated in the whole process of research. $\mathrm{CL}$ and $\mathrm{LL}$ conducted literature screening and data extraction, $\mathrm{YH}$ was responsible for data analysis and interpretation. $\mathrm{CL}, \mathrm{YH}$ and $\mathrm{LL}$ wrote this manuscript collaboratively, FL and YS contributed to the final revision of the paper. All authors have read and approved the final manuscript.

\section{Funding}

None.

\section{Availability of data and materials}

Data sharing is not applicable to this article as no datasets were generated or analysed during the current study.

Ethics approval and consent to participate Not applicable.

\section{Consent for publication}

Not applicable.

Competing interests

Yuan Shi is an Associate Editor in this journal. 
Received: 18 May 2020 Accepted: 24 August 2020 Published online: 01 September 2020

\section{References}

1. Wu F, Zhao S, Yu B, Chen YM, Wang W, et al. A new coronavirus associated with human respiratory disease in China. Nature. 2020;579(7798):265-9.

2. Jiang S, Shi Z, Shu Y, Song J, Gao GF, Tan W, et al. A distinct name is needed for the new coronavirus. Lancet. 2020;395:949.

3. Chan JF-W, Yuan S, Kok K-H, To KKW, Chu H, Yang J, et al. A familial cluster of pneumonia associated with the 2019 novel coronavirus indicating person-to-person transmission: a study of a family cluster. Lancet. 2020;395: 514-23.

4. Coronavirus disease 2019 (COVID-19). Situation Report Situation Report -90. 2020. https://www.who.int/docs/default-source/coronaviruse/situationreports/20200517-sitrep-118-covid-19.pdf. Accessed 17 May 2020.

5. Lu R, Zhao X, Li J, Niu P, Yang B, Wu H, et al. Genomic characterisation and epidemiology of 2019 novel coronavirus: implications for virus origins and receptor binding. Lancet. 2020;395:565-74.

6. Novel C P E R E. The epidemiological characteristics of an outbreak of 2019 novel coronavirus diseases (COVID-19) in China. Chin J Epidemiol. 2020;41: 145. https://doi.org/10.3760/cma.j.jissn.0254-6450.2020.02.003.

7. Li AM, Ng PC. Severe acute respiratory syndrome (SARS) in neonates and children. Arch Dis Child Fetal Neonatal Ed. 2005;90:F461-5.

8. Al-Tawfiq JA, Kattan RF, Memish ZA. Middle East respiratory syndrome coronavirus disease is rare in children: an update from Saudi Arabia. World J Clin Pediatr. 2016;5:391-6.

9. Dong Y, Mo X, Hu Y, et al. Epidemiological characteristics of 2143 pediatric patients with 2019 coronavirus disease in China. Pediatrics. 2020:e20200702.

10. Moher D, Liberati A, Tetzlaff J, Altman DG, Group P. Preferred reporting items for systematic reviews and meta-analyses: the PRISMA statement. J Clin Epidemiol. 2009;62:1006-12.

11. Moga C, Guo B, Schopflocher D, Harstall C. Development of a Quality Appraisal Tool for Case Series Studies Using a Modified Delphi Technique. Edmonton AB: Institute of Health Economics; 2012.

12. Zhou $Y$, Yang GD, Feng $K$, Huang $H$, Yun YX, Mou XY, et al. Clinical features and chest CT findings of coronavirus disease 2019 in infants and young children. CJCP. 2020;22:215-20. https://doi.org/10.7499/j.issn.1008-8830.2020. 03.007 .

13. Liu W, Zhang Q, Chen J, et al. Detection of Covid-19 in children in early January 2020 in Wuhan, China. N Engl J Med. 2020;382:1370-1.

14. Wang D, Ju XL, Xie F, Lu Y, Li FY, Huang HH, et al. Clinical analysis of 31 cases of 2019 novel coronavirus infection in children from six provinces (autonomous region) of northern China. Chin J Pediatr. 2020;58:E011. https://doi.org/10.3760/cma.j.cn112140-20200225-00138.

15. Hu Z, Song C, Xu C, Jin G, Chen Y, Xu X, et al. Clinical characteristics of 24 asymptomatic infections with COVID-19 screened among close contacts in Nanjing, China. Sci China Life Sci. 2020;63:706-11.

16. Xu Y, Li X, Zhu B, Liang H, Fang C, Gong Y, et al. Characteristics of pediatric SARS-CoV-2 infection and potential evidence for persistent fecal viral shedding. Nat Med. 2020:26(4):502-5.

17. Cai J, Xu J, Lin D, Yang Z, Xu L, Qu Z, et al. A case series of children with 2019 novel coronavirus infection: clinical and epidemical features. Clin Infect Dis. 2020. https://doi.org/10.1093/cid/ciaa198.

18. Lu X, Zhang L, Du H, Zhang J, Li Y, Qu J, et al. SARS-CoV-2 infection in children. N Engl J Med. 2020;382:1663-5.

19. Qiu HY, Wu J, Hong L, Luo Y, Song Q, Chen D. Clinical and epidemiological features of 36 children with coronavirus disease 2019 (COVID-19) in Zhejiang, China: an observational cohort study. Lancet Infect Dis. 2020. https://doi.org/10.1016/S1473-3099(20)30198-5.

20. Li W, Cui H, Li K, Fang Y, Li S. Chest computed tomography in children with COVID-19 respiratory infection. Pediatr Radiol. 2020. https://doi.org/10.1007/ s00247-020-04656-7.

21. Feng $K$, Yun YX, Wang XF, Yang GD, Zheng YJ, Lin CM, et al. Analysis of CT features of 15 children with 2019 novel coronavirus infection. Chin J Pediatr 2020;58(0): E007; doi: https://doi.org/10.3760/cma.j.cn112140-2020021000071.

22. Wei M, Yuan J, Liu Y, Fu T, Yu X, Zhang ZJ. Novel coronavirus infection in hospitalized infants under 1 year of age in China. JAMA. 2020;323:1313-4.

23. Zhong Z, Xie XZ, Huang W, Zhao W, Yu QZ, Liu J. Chest CT findings and clinical features of coronavirus disease 2019 in children. J Cent South Univ
(Med Sci). 2020;45:236-42. https://doi.org/10.11817/j.issn.1672-7347.2020 200206.

24. Ma X, Liang S, Zhang YK, Zhang XZ, Gai ZT, Zhang ZF. Do children need a longer time to shed SARS-CoV-2 in stool than adults? J Microbiol Immunol Infect. 2020;53(3):373-6.

25. Tan X, Huang J, Zhao F, Zhou Y, Li JQ, Wang XY. Clinical features of children with SARS-CoV-2 infection: an analysis of 13 cases from Changsha, China. Chin J Contemp Pediatr. 2020;22(4):294.

26. Zhu L, Wang J, Huang R, Liu L, Zhao H, Wu C, et al. Clinical characteristics of a case series of children with coronavirus disease 2019. Pediatr Pulmonol. 2020;55(6):1430-2.

27. Tan YP, Tan BY, Pan J, Wu J, Zeng SZ, Wei HY. Epidemiologic and clinical characteristics of 10 children with coronavirus disease 2019 in Changsha, China. J Clin Virol. 2020;104353. https://doi.org/10.1016/j.jcv.2020.104353.

28. Chen J, Zhang ZZ, Chen YK, Long QX, Tian WG, Deng HJ, et al. The clinical and immunological features of pediatric COVID-19 patients in China. Genes Dis. 2020. https://doi.org/10.1016/j.gendis.2020.03.008.

29. Song $R$, Han B, Song M, Wang L, Conlon CP, Dong T, et al. Clinical and epidemiological features of COVID-19 family clusters in Beijing, China. J Inf Secur. 2020;81(2):e26-30. https://doi.org/10.1016/j.jinf.2020.04.018.

30. Song W, Li J, Zou N, Guan W, Pan J, Xu W. Clinical features of pediatric patients with coronavirus disease (COVID-19). J Clin Virol. 2020;104377. https://doi.org/10.1016/j.jcv.2020.104377.

31. Wu HP, Li BF, Chen X, Hu HZ, Jiang SA, Cheng H. Clinical features of coronavirus disease 2019 in children aged $<18$ years in Jiangxi, China: an analysis of 23 cases. Chin J Contemp Pediatr. 2020;22(5):419-24. https://doi. org/10.7499/j.issn.1008-8830.2003202.

32. Parri N, Lenge M, Buonsenso D. Children with Covid-19 in pediatric emergency departments in Italy. N Engl J Med. 2020;383(2):187-90.

33. Musolino AM, Supino MC, Buonsenso D, Ferro V, Valentini P, Magistrelli A et al. Lung ultrasound in children with COVID-19: preliminary findings. Ultrasound Med Biol. 2020;46(8):2094-8.

34. Zhang B, Liu S, Zhang J, Xiao J, Zhu S, Dong Y, et al. Children hospitalized for coronavirus disease 2019 (COVID-19): a multicenter retrospective descriptive study. J Inf Secur. 2020;81(2):e74-5. https://doi.org/10.1016/j.jinf. 2020.04.045.

35. Garazzino S, Montagnani C, Donà D, Meini A, Felici E, Vergine G, et al. Multicentre Italian study of SARS-CoV-2 infection in children and adolescents, preliminary data as at 10 April 2020. Euro Surveill. 2020. https:// doi.org/10.2807/1560-7917.ES.2020.25.18.2000600.

36. de Rojas T, Pérez-Martínez A, Cela E, Baragaño M, Galán V, Mata C, et al. COVID-19 infection in children and adolescents with cancer in Madrid. Pediatr Blood Cancer. 2020;67(7):e28397.

37. Paquette D, Bell C, Roy M, Whitmore L, Currie A, Archibald C, et al. Laboratory-confirmed COVID-19 in children and youth in Canada, January 15-April 27, 2020. Can Commun Dis Rep. 2020;46(5):121-4.

38. CDC COVID-19 Response Team. Coronavirus Disease 2019 in Children United States, February 12-April 2, 2020. MMWR Morb Mortal Wkly Rep. 2020;69(14):422-6.

39. Li B, Shen J, Li L, Yu C. Radiographic and clinical features of children with Coronavirus disease (COVID-19) pneumonia. Indian Pediatr. 2020; 57(5):423-6

40. Li $Y$, Ye $Y$, Xuan $W$, Chen $Y, W u$ B, Chen Z. Imaging features of the initial chest high resolution CT scan in juvenile patient with Coronavirus disease 2019. Chin J Gen Pract. 2020;19. https://doi.org/10.3760/cma.j. cn11479820200216-00117.

41. Rodriguez-Morales AJ, Cardona-Ospina JA, Gutiérrez-Ocampo E, VillamizarPeña R, Holguin-Rivera Y, Escalera-Antezana JP, et al. Clinical, laboratory and imaging features of COVID-19: a systematic review and meta-analysis. Travel Med Infect Dis. 2020. https://doi.org/10.1016/j.tmaid.2020.101623.

42. Leung $C$, Kwan $Y$, Ko $P$, et al. Severe acute respiratory syndrome among children. Pediatrics. 2004;113:e535-43.

43. Zheng Y, He Y, Deng J, Lu Z, Wei J, Yang W, et al. Hospitalized children with 2009 influenza a (H1N1) infection in Shenzhen, China, NovemberDecember 2009. Pediatr Pulmonol. 2011;46:246-52.

44. Cao Q, Chen YC, Chen CL, Chiu CH. SARS-CoV-2 infection in children: transmission dynamics and clinical characteristics. J Formos Med Assoc. 2020;119:670-3.

45. Henry BM, Lippi G, Plebani M. Laboratory abnormalities in children with novel coronavirus disease 2019. CCLM. 2020. https://doi.org/10.1515/cclm2020-0272. 
46. Rodríguez-Morales AJ, MacGregor K, Kanagarajah S, Patel D, Schlagenhauf P. Going global-travel and the 2019 novel coronavirus. Travel Med Infect Dis. 2020. https://doi.org/10.1016/j.tmaid.2020.101578.

47. Zhou $P$, Yang XL, Wang XG, Hu B, Zhang L, Zhang W, et al. A pneumonia outbreak associated with a new coronavirus of probable bat origin. Nature. 2020;579:270-3.

48. Wang D, Hu B, Hu C, Zhu F, Liu X, Zhang J, et al. Clinical characteristics of 138 hospitalized patients with 2019 Novel Coronavirus-infected pneumonia in Wuhan, China. JAMA. 2020;323(11):1061-9.

49. Update on COVID-19 outbreak as of 24:00 on 17 May. http://www.chinacdc. cn/jkzt/crb/zl/szkb_11803/jszl_11809/202005/t20200518_216755.html Accessed 17 May 2020

50. Leung K, Wu JT, Liu D, Leu GM. First-wave COVID-19 transmissibility and severity in China outside Hubei after control measures, and second-wave scenario planning: a modelling impact assessment. Lancet. 2020;395:1382-93.

51. Xu S, Li Y. Beware of the second wave of COVID-19. Lancet. 2020;395:1321-2.

52. Viner RM, Russell SJ, Croker H, MEpi JP, MBBS JW, Stansfield C, et al. School closure and management practices during coronavirus outbreaks including COVID-19: a rapid systematic review. Lancet Child Adolesc Health. 2020;5: 397-404.

53. Bayham J, Fenichel EP. Impact of school closures for COVID-19 on the US health-care workforce and net mortality: a modelling study. Lancet Public Health. 2020. https://doi.org/10.1016/S2468-2667(20)30082-7.

54. Guidance-What parents and carers need to know about schools and education during the coronavirus outbreak. https://www.gov.uk/ government/publications/closure-of-educational-settings-information-forparents-and-carers/closure-of-educational-settings-information-for-parentsand-carers\#closures-of-schools-childcare-and-other-educational-settings. Accessed 17 May 2020.

55. Interim Guidance for Administrators of US K-12 Schools and Child Care Programs to Plan, Prepare, and Respond to Coronavirus Disease 2019 (COVID-19). https://www.cdc.gov/coronavirus/2019-ncov/community/ schools-childcare/guidance-for-schools-h.pdf. Accessed 17 May 2020.

56. Coronavirus, the measures taken by the Government. http://www.governo it/it/coronavirus-misure-del-governo Accessed 17 May 2020.

57. Informations Coronavirus. https://www.gouvernement.fr/info-coronavirus. Accessed 17 May 2020.

58. Coronavirus in Deutschland. https://www.bundesregierung.de/breg-de/ suche/992800!search?f=1495774\%3A1726012. Accessed 17 May 2020.

59. The Lancet Child Adolescent, H. Pandemic school closures: risks and opportunities. Lancet Child Adolesc Health. 2020. https://doi.org/10.1016/ S2352-4642(20)30105-X.

60. Wang G, Zhang Y, Zhao J, Zhang J, Jiang F. Mitigate the effects of home confinement on children during the COVID-19 outbreak. Lancet. 2020;395:945-7.

\section{Publisher's Note}

Springer Nature remains neutral with regard to jurisdictional claims in published maps and institutional affiliations.

Ready to submit your research? Choose BMC and benefit from:

- fast, convenient online submission

- thorough peer review by experienced researchers in your field

- rapid publication on acceptance

- support for research data, including large and complex data types

- gold Open Access which fosters wider collaboration and increased citations

- maximum visibility for your research: over $100 \mathrm{M}$ website views per year

At BMC, research is always in progress.

Learn more biomedcentral.com/submissions 